\title{
Natural Convection Flow along an Isothermal Vertical Flat Plate with Temperature Dependent Viscosity and Heat Generation
}

\author{
Md. Mamun Molla, ${ }^{1}$ Anita Biswas, ${ }^{2}$ Abdullah Al-Mamun, ${ }^{3}$ and Md. Anwar Hossain ${ }^{4}$ \\ ${ }^{1}$ School of Engineering \& Applied Science, Department of Electrical \& Computer Engineering, \\ North South University, Dhaka 1229, Bangladesh \\ ${ }^{2}$ Department of Mathematics, Jahangirnagar University, Dhaka, Bangladesh \\ ${ }^{3}$ Institute of Natural Sciences, United International University, Dhaka 1209, Bangladesh \\ ${ }^{4}$ Department of Mathematics, University of Dhaka, Dhaka 1000, Bangladesh
}

Correspondence should be addressed to Md. Mamun Molla; mmamun@northsouth.edu

Received 12 November 2013; Revised 23 April 2014; Accepted 23 April 2014; Published 27 May 2014

Academic Editor: Clement Kleinstreuer

Copyright (C) $2014 \mathrm{Md}$. Mamun Molla et al. This is an open access article distributed under the Creative Commons Attribution License, which permits unrestricted use, distribution, and reproduction in any medium, provided the original work is properly cited.

The purpose of this study is to investigate the natural convection laminar flow along an isothermal vertical flat plate immersed in a fluid with viscosity which is the exponential function of fluid temperature in presence of internal heat generation. The governing boundary layer equations are transformed into a nondimensional form and the resulting nonlinear system of partial differential equations is reduced to a convenient form which are solved numerically using an efficient marching order implicit finite difference method with double sweep technique. Numerical results are presented in terms of the velocity and temperature distribution of the fluid as well as the heat transfer characteristics, namely, the wall shear stress and the local and average rate of heat transfer in terms of the local skin-friction coefficient, the local and average Nusselt number for a wide range of the viscosity-variation parameter, heat generation parameter, and the Rayleigh number. Increasing viscosity variation parameter and Rayleigh number lead to increasing the local and average Nusselt number and decreasing the wall shear stress. Wall shear stress and the rate of heat transfer decreased due to the increase of heat generation.

\section{Introduction}

A large number of physical phenomena involve natural convection driven by heat generation. The study of heat generation in moving fluids is important in view of several physical problems such as those dealing with chemical reactions and those concerned with dissociating fluids. Possible heat generation effects may alter the temperature distribution and, therefore, the particle deposition rate. This may occur in such applications related to nuclear reactor cores, fire and combustion modeling, electronic chips, and semiconductor wafers. In fact, the literature is replete with examples dealing with the heat transfer in laminar flow of viscous fluids. Vajravelu and Hadjinicolaou [1] studied the heat transfer characteristics in the laminar boundary layer of a viscous fluid over a linearly stretching continuous surface with viscous dissipation or frictional heating and internal heat generation. In this study, Vajravelu and Hadjinicolaou [1] considered that the volumetric rate of heat generation, $q^{\prime \prime}$ $\left[\mathrm{W} / \mathrm{m}^{3}\right]$, should be

$$
q^{\prime \prime}= \begin{cases}Q_{0}\left(\bar{T}-T_{\infty}\right), & \text { for } \bar{T} \geq T_{\infty}, \\ 0, & \text { for } \bar{T}<T_{\infty}\end{cases}
$$

where $Q_{0}$ is the heat generation constant. The above relation, explained by Vajravelu and Hadjinicolaou [1], is valid as an approximation of the state of some exothermic process and having $T_{\infty}$ as the onset temperature. Following Vajravelu and Hadjinicolaou [1], Molla et al. [2-5] investigated the natural convection with heat generation along a vertical wavy surface, horizontal circular cylinder, and sphere, respectively. 
In all of the above studies, the authors assumed that the viscosities of the fluids are constant throughout the flow regime. But this physical property may change significantly with temperature. For example, the viscosity of air is $0.6924 \times$ $10^{-5} \mathrm{~kg} / \mathrm{m} \cdot \mathrm{s}, 1.3289 \mathrm{~kg} / \mathrm{m} \cdot \mathrm{s}, 2.286 \mathrm{~kg} / \mathrm{m} \cdot \mathrm{s}$, and $3.625 \mathrm{~kg} / \mathrm{m} \cdot \mathrm{s}$ at $100 \mathrm{~K}, 200 \mathrm{~K}, 400 \mathrm{~K}$, and $800 \mathrm{~K}$ temperature, respectively. (See Cebeci and Bradshaw [6].) In order to predict accurately the flow behaviour, it is necessary to take into account the temperature dependence of viscosity. Gary et al. [7] and Mehta and Sood [8] found that the flow characteristics change substantially when the effect of temperatur-dependent viscosity was considered. Hady et al. [9] have also investigated the mixed convection boundary layer flow on a continuous flat plate with variable viscosity. Kafoussias and Williams [10] and Kafoussias et al. [11] have studied the effects of variable viscosity on the free and mixed convection flow from a vertical flat plate in the region near the leading edge. Hossain et al. [12, 13] and Molla et al. [14] considered the natural convection with variable viscosity, where the viscosity is inversely proportional to a linear function of temperature, a model that was proposed by Ling and Dybbs [15]. Besides these, it has been found that for liquid, such as organ gas, the viscosity varies with temperature in an approximately linear manner. On assuming that the viscosity of the fluid is linear functions of temperature, a semiempirical formula was proposed by Charraudeau [16]. Following him Molla et al. $[17,18]$ have investigated the effects of chemical reaction, heat, and mass diffusion on natural convection flow from an isothermal sphere and circular cylinder with temperaturedependent viscosity.

Natural convection flows were analyzed applying a new method in order to obtain the approximate equations by Gray and Giorgini [19]. The established Boussinesq approximation was verified under this method in the case of a given Newtonian liquid or gas. Considering the constant property (CP) model and the variable property (VP) model the laminar natural convection flow along a horizontal rectangular duct taking into account the significant buoyancy effects was studied by Wang et al. [20]. The Boussinesq approximations were introduced for the CP model and the properties were used at four different reference temperatures. On the other hand, the density and the transport properties were worked out with the help of the state equation of an ideal gas and power law correlations.

Saha et al. [21] investigated the natural convection flow along an isothermal vertical plate surrounded in a stratified medium with uniform heat source. The numerical solutions of the nonsimilar equations were carried out by the implicit finite difference method and it is mentioned that the same method will be used in the present investigation. The nonsimilar laminar free convection adjacent to a vertical wall applying exceptional prescribed boundary conditions was analyzed by Kao [22]. The locally nonsimilar method for the second order truncation was employed in the study in order to investigate the nonsimilar free convection problems. The numerical simulations of the buoyancy forced flow over an isothermal vertical surface having a leading edge were carried out by Wright and Gebhart [23]. In this study the coordinate transformations were taken under consideration in order to allow efficient calculation of both the boundary layer flow and the extensive ambient entrainment in-flow.

But thermal convection in a fluid whose viscosity is strongly temperature dependent is of interest in geophysical problems as well as in various engineering applications. Numerous investigations have been focused on theoretical and experimental aspects of this problem, such as those of Torrance and Turcotte [24], Booker [25], Stengel et al. [26], Richter et al. [27], and Bottaro et al. [28]. Torrance and Turcotte [24] have investigated the influence of large variations of viscosity on convection in a layer of fluid heated from below. Solutions for the flow and temperature fields were obtained numerically assuming infinite Prandtl number, freesurface boundary conditions, and two-dimensional motion of fixed horizontal wavelength. The effects of a temperaturedependent and a depth-dependent viscosity were each studied. Thermal convection in a fluid with viscosity which is strong function of temperature may be directly applicable to the earth's mantle. So, it is known that the viscosity of the mantle can be formulated as the exponential function of temperature.

The effect of the heat generation on natural convection flow along a vertical flat plate with the exponentially varying temperature-dependent kinematic viscosity has not been studied yet and the present work demonstrates the issue. For the experimentalists, this investigating numerical result helps a lot to predict the flow behaviour. In this investigation the focus is on the boundary layer regime promoted by the combined events along a flat plate with temperaturedependent viscosity $\Pi(T)$ (as in Torrance and Turcotte [24]) in presence of heat generation while the surface is at a uniform temperature. The basic equations are transformed to the convenient form of the boundary layer equations, which are solved numerically using a very efficient marching order implicit finite-difference method. Consideration is given to the situation where the buoyancy forces assist the natural convection flow for various combinations of the viscosityvariation parameter $\varepsilon$, heat generation parameter $\lambda$, and the Rayleigh number Ra. The results allow us to predict the different behavior that can be observed when the relevant parameters are varied.

\section{Formulation of Problem}

Consider a steady two-dimensional laminar natural convective flow from a uniformly heated semi-infinite vertical flat plate, which is immersed in a viscous and incompressible fluid having temperature-dependent kinematic viscosity. Here kinematic viscosity is the exponential function of the fluid temperature. It is assumed that the surface temperature of the flat plate is $T_{w}$, where $T_{w}>T_{\infty}$. Here $T_{\infty}$ is the ambient temperature of the fluid; the configuration considered is as shown in Figure 1. In Figure 1, $\delta_{M}$ and $\delta_{T}$ represent the momentum and thermal boundary layer thickness, respectively. 


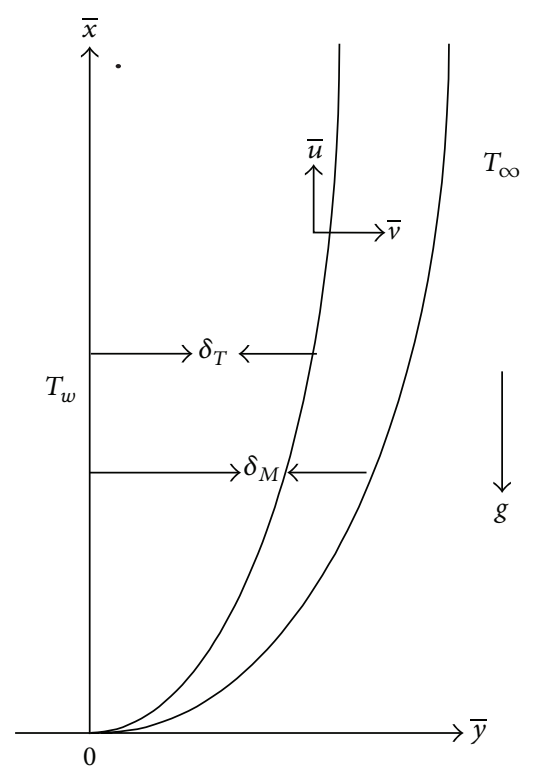

FIgURE 1: Physical model and coordinate system.

The equations governing the flow are

$$
\begin{gathered}
\frac{\partial \bar{u}}{\partial \bar{x}}+\frac{\partial \bar{v}}{\partial \bar{y}}=0 \\
\bar{u} \frac{\partial \bar{u}}{\partial \bar{x}}+\bar{v} \frac{\partial \bar{u}}{\partial \bar{y}}=\frac{\partial}{\partial \bar{y}}\left(\nu \frac{\partial \bar{u}}{\partial \bar{y}}\right)+g \beta\left(\bar{T}-T_{\infty}\right), \\
\bar{u} \frac{\partial \bar{T}}{\partial \bar{x}}+\bar{v} \frac{\partial \bar{T}}{\partial \bar{y}}=\alpha \frac{\partial^{2} \bar{T}}{\partial \bar{y}^{2}}+\frac{Q_{0}}{\rho C_{p}}\left(\bar{T}-T_{\infty}\right) .
\end{gathered}
$$

The boundary conditions of (2) are

$$
\begin{gathered}
\bar{u}=\bar{v}=0, \quad \bar{T}=T_{w}, \quad \text { at } \bar{y}=0, \\
\bar{u} \longrightarrow 0, \quad \bar{T} \longrightarrow T_{\infty} \quad \text { as } \bar{y} \longrightarrow \infty,
\end{gathered}
$$

where $(\bar{u}, \bar{v})$ are velocity components along the $(\bar{x}, \bar{y})$ axes, $g$ is the acceleration due to gravity, $\rho$ is the density, $v$ is the dimensional kinematic viscosity of the fluid depending on the fluid temperature $\bar{T}, \beta$ is the coefficient of thermal expansion, $\alpha$ is the thermal diffusivity of the fluid, and $Q_{0}$ being a constant, which may take either positive or negative. The source term represents the heat generation when $Q_{0}>0$ and the heat absorption when $Q_{0}<0$ and $C_{p}$ is the specific heat at constant pressure.
We now introduce the following nondimensional variables:

$$
\begin{gathered}
x=\frac{\bar{x}}{l}, \quad y=\frac{\bar{y}}{l}, \quad u=\frac{l}{\alpha} \bar{u}, \\
v=\frac{l}{\alpha} \bar{v}, \quad \theta=\frac{\bar{T}-T_{\infty}}{T_{w}-T_{\infty}}, \quad \Pi=\frac{\nu}{\nu_{0}} \\
\operatorname{Ra}=\frac{g \beta\left(T_{w}-T_{\infty}\right) l^{3}}{\alpha \nu_{0}}=\mathrm{Gr} \operatorname{Pr}, \\
\operatorname{Pr}=\frac{\nu_{0}}{\alpha}, \quad \mathrm{Gr}=\frac{g \beta\left(T_{w}-T_{\infty}\right) l^{3}}{\nu_{0}^{2}},
\end{gathered}
$$

where $v_{0}$ is the reference kinematic viscosity at the ambient, $l$ is the characteristic length of the vertical flat plate, Gr is the Grashof number, $\mathrm{Pr}$ is the Prandtl number, $\mathrm{Ra}$ is the Rayleigh number, and $T$ is the nondimensional temperature.

Out of the many forms of kinematic viscosity variation, which are available in the literature, we will consider only the following form proposed by Torrance and Turcotte [24]:

$$
\nu=v_{0} \exp \left[\varepsilon\left(\frac{1}{2}-\frac{\bar{T}-T_{\infty}}{T_{w}-T_{\infty}}\right)\right],
$$

where $\varepsilon$ is the viscosity-variation parameter. When $\varepsilon=0.0$, the kinematic viscosity of the fluid is constant. If $\varepsilon<0.0$, the kinematic viscosity is larger than the constant kinematic viscosity and if $\varepsilon>0.0$, the kinematic viscosity is smaller than the case of $\varepsilon=0.0$.

Substituting variables (4) into (2) leads to the following nondimensional equations:

$$
\begin{gathered}
\frac{\partial u}{\partial x}+\frac{\partial v}{\partial y}=0 \\
u \frac{\partial u}{\partial x}+v \frac{\partial u}{\partial y}=\operatorname{Pr} \Pi\left[\frac{\partial^{2} u}{\partial y^{2}}-\varepsilon \frac{\partial u}{\partial y} \frac{\partial T}{\partial y}\right]+\operatorname{Ra} \operatorname{Pr} \theta \\
u \frac{\partial \theta}{\partial x}+v \frac{\partial \theta}{\partial y}=\frac{\partial^{2} \theta}{\partial y^{2}}+\lambda \theta
\end{gathered}
$$

where $\Pi$ is the form of variable kinematic viscosity and $\lambda$ is the heat generation parameter defined, respectively, as

$$
\Pi=\exp \left[\varepsilon\left(\frac{1}{2}-\theta\right)\right], \quad \lambda=\frac{Q_{0} l^{2}}{\rho \alpha C_{p}} .
$$

The boundary conditions (3a) and (3b) become

$$
\begin{gathered}
u=v=0, \quad \theta=1 \quad \text { at } y=0, \\
u \longrightarrow 0, \quad \theta \longrightarrow 0 \quad \text { as } y \longrightarrow \infty .
\end{gathered}
$$

Here we introduce new transformations for the numerical scheme

$$
\begin{gathered}
X=x, \quad Y=\frac{y}{x^{1 / 4}}, \quad U=\frac{u}{x^{1 / 2}} \\
V=x^{1 / 4} v .
\end{gathered}
$$




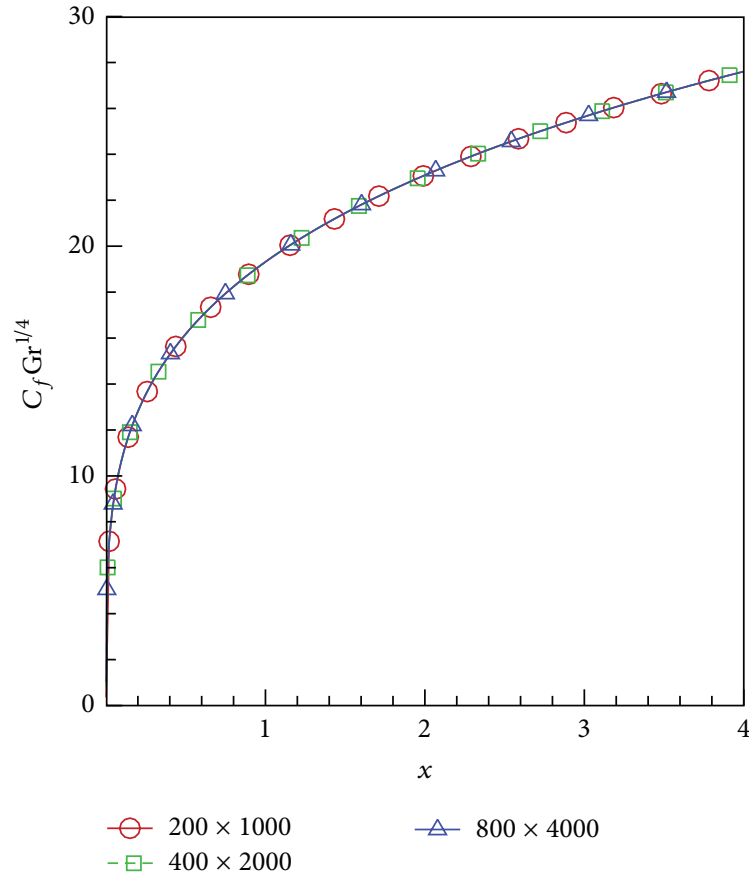

(a)

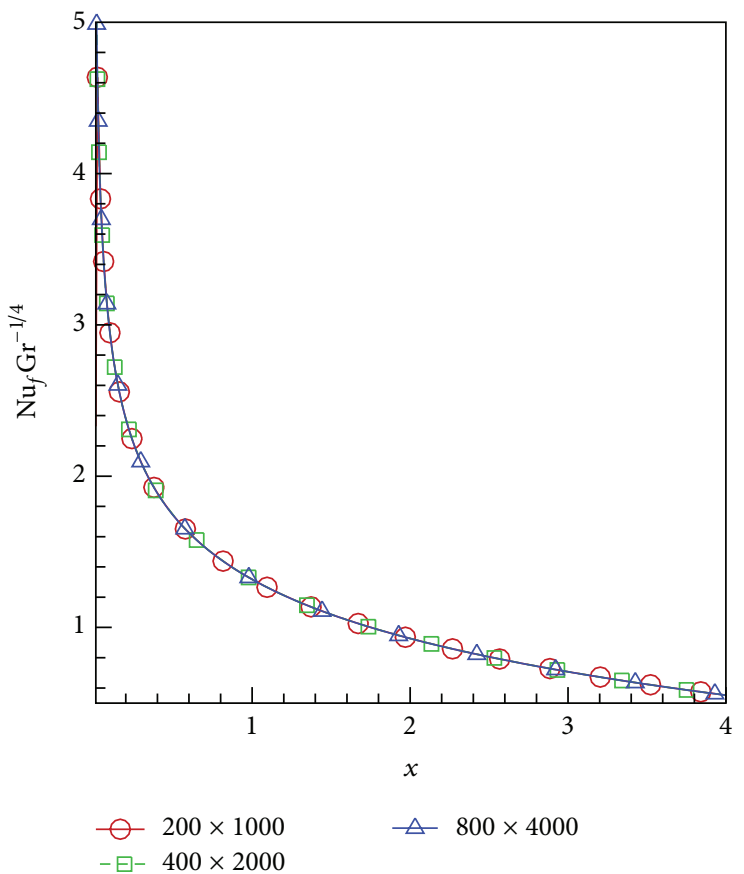

(b)

FIGURE 2: Grid independence test in terms of (a) the local skin-friction coefficient and (b) Nusselt number while $\operatorname{Pr}=1.0, \mathrm{Ra}=200, \varepsilon=5$, and $\lambda=2.5$.

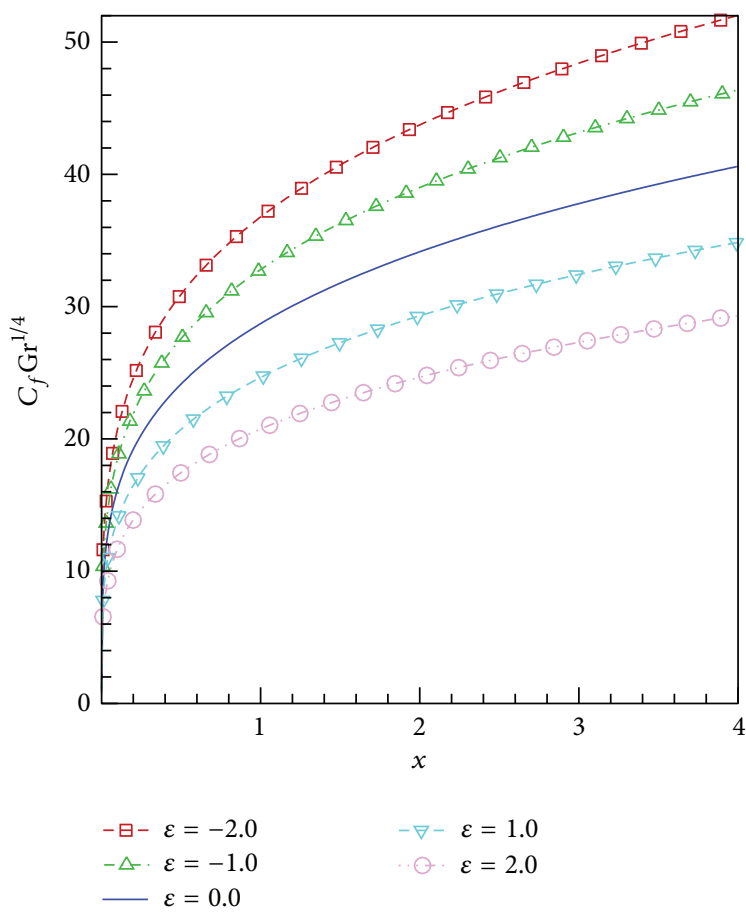

(a)

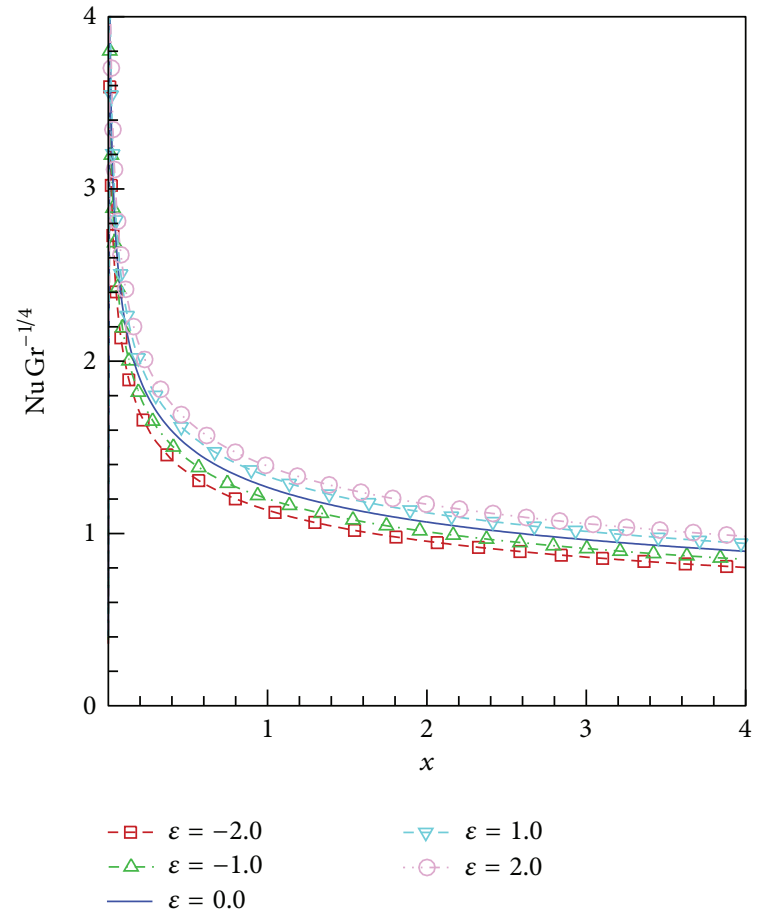

(b)

FIgURE 3: (a) Skin-friction coefficient. (b) Rate of heat transfer for different values of $\varepsilon$ while $\mathrm{Ra}=100.0$ and $\lambda=0.0$. 


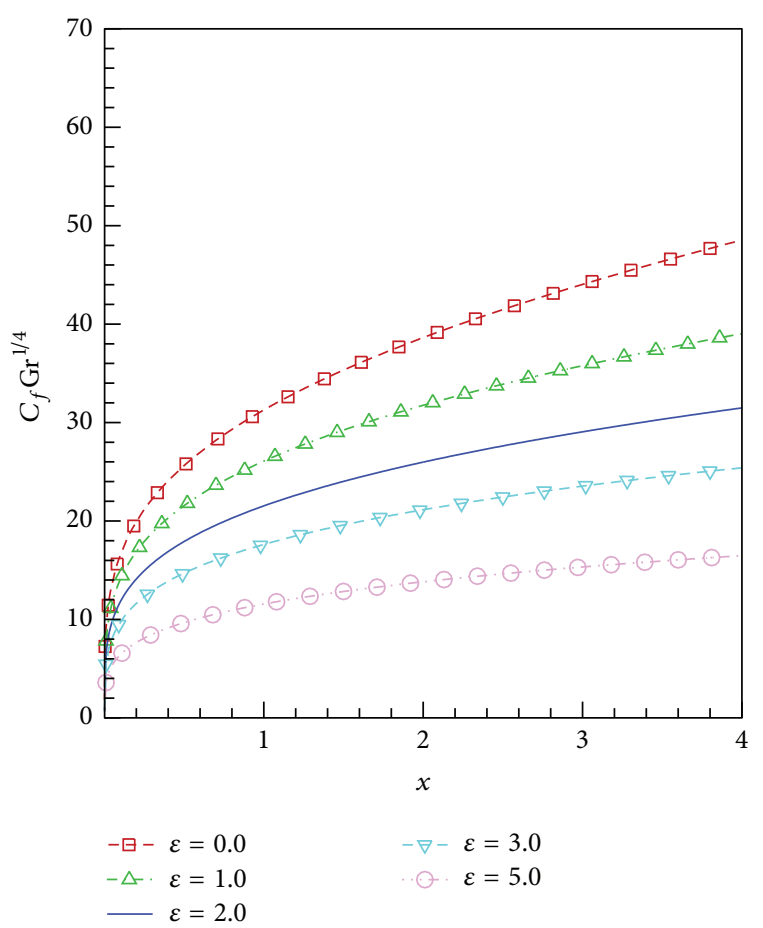

(a)

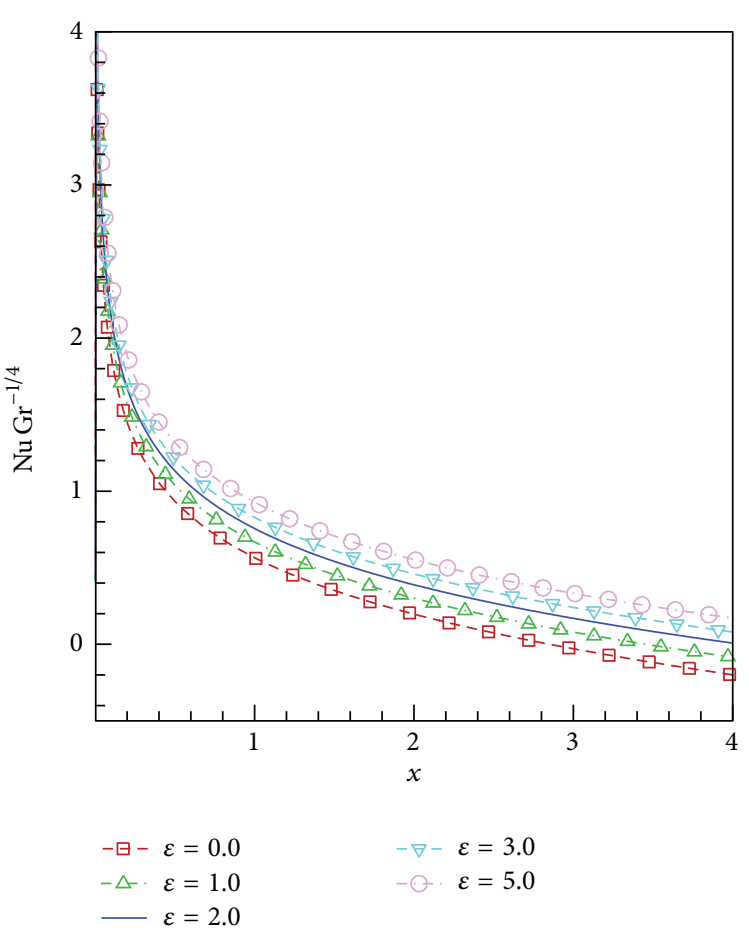

(b)

Figure 4: (a) Skin-friction coefficient. (b) Rate of heat transfer for different values of $\varepsilon$ while $\operatorname{Ra}=100.0$ and $\lambda=2.5$.

Using (9) into (6), we get

$$
\begin{gathered}
X \frac{\partial U}{\partial X}+\frac{1}{2} U-\frac{1}{4} Y \frac{\partial U}{\partial Y}+\frac{\partial V}{\partial Y}=0, \\
X U \frac{\partial U}{\partial X}+\left(V-\frac{1}{4} Y U\right) \frac{\partial U}{\partial Y}+\frac{1}{2} U^{2} \\
=\operatorname{Pr}\left[\frac{\partial^{2} U}{\partial Y^{2}}-\varepsilon \frac{\partial U}{\partial Y} \frac{\partial \theta}{\partial Y}\right]+\operatorname{Ra} \operatorname{Pr} \theta, \\
X U \frac{\partial \theta}{\partial X}+\left(V-\frac{1}{4} Y U\right) \frac{\partial \theta}{\partial Y}=\frac{\partial^{2} \theta}{\partial Y^{2}}+X^{1 / 2} \lambda \theta .
\end{gathered}
$$

The corresponding boundary conditions are

$$
\begin{gathered}
U=V=0, \quad \theta=1 \quad \text { at } Y=0, \\
U \longrightarrow 0, \quad \theta \longrightarrow 0 \quad \text { as } Y \longrightarrow \infty .
\end{gathered}
$$

Now (10)-(12) subject to the boundary conditions (13a) and (13b) are discretised for numerical scheme using centraldifference for diffusion terms and the forward-difference for the convection terms; finally we get a system of tridiagonal algebraic equations. The algebraic equations have been solved by Gaussian elimination technique. In computation we directly solve the continuity equation for the normal velocity $V$ by the following discretisation:

$$
\begin{aligned}
V_{i, j}= & V_{i, j-1}+Y_{j} \frac{1}{4}\left(U_{i, j}-U_{i, j-1}\right) \\
& -\Delta Y \frac{1}{4}\left(U_{i, j-1}+U_{i, j}\right)-X_{i} \frac{\Delta Y}{\Delta X}\left(U_{i, j}-U_{i-1, j}\right) .
\end{aligned}
$$

The computation is started at $X=0.0$ and then marches up to the point $(X=4.0)$. Here $\Delta x=\Delta y=0.01$ are used for the $X$ - and $Y$-grids, respectively. The physical quantities, namely, the local skin-friction coefficient and the local Nusselt number which are important from the application point of view, are calculated from the following dimensionless relations:

$$
\begin{gathered}
C_{f} \mathrm{Gr}^{1 / 4}=\exp \left(-\frac{\varepsilon}{2}\right) X^{1 / 4}\left(\frac{\partial U}{\partial Y}\right)_{Y=0}, \\
\mathrm{Nu} \mathrm{Gr}^{-1 / 4}=-X^{-1 / 4}\left(\frac{\partial \theta}{\partial Y}\right)_{Y=0} .
\end{gathered}
$$

For calculating the stream function $\Psi$, the fluid velocity over the whole boundary layer has been integrated, which can be written as

$$
\Psi=\int_{0}^{Y} X^{1 / 2} U d Y .
$$

The average Nusselt number is calculated from the following relation:

$$
\mathrm{Nu}_{m} \mathrm{Gr}^{-1 / 4}=-\frac{1}{l} \int_{0}^{l} X^{-1 / 4}\left(\frac{\partial \theta}{\partial Y}\right)_{Y=0} d x .
$$




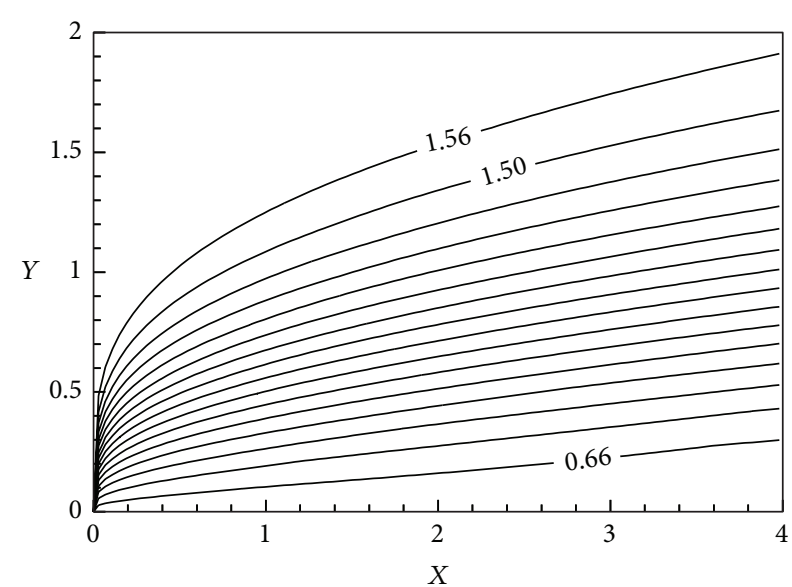

(a)

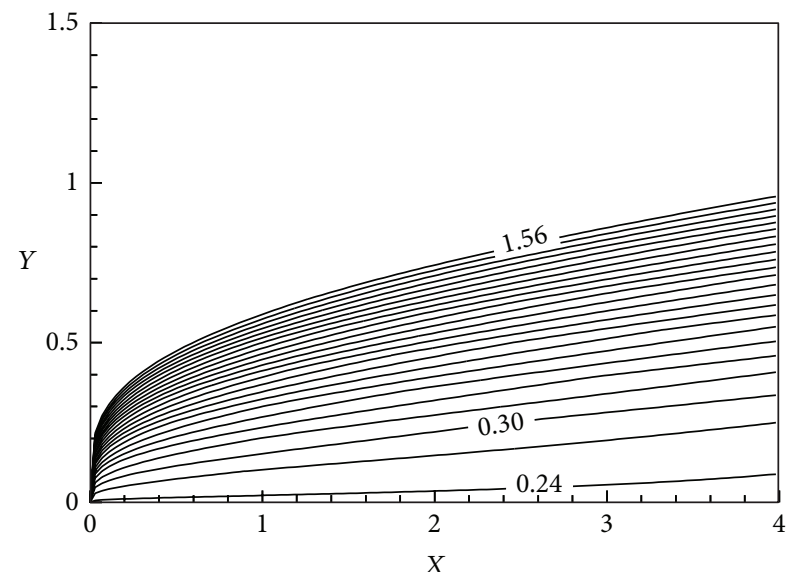

(b)

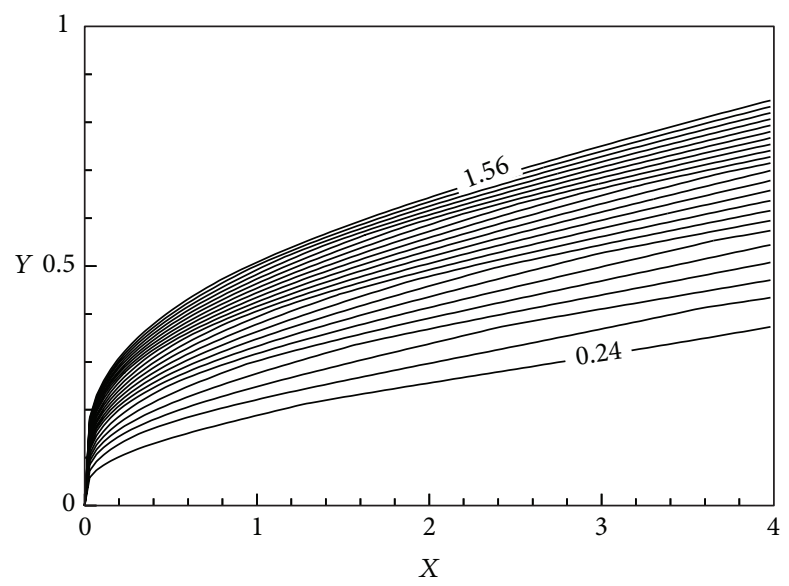

(c)

Figure 5: Isolines viscosity for (a) $\varepsilon=1.0$, (b) $\varepsilon=3.0$, and (c) $\varepsilon=5.0$ while $\mathrm{Ra}=100.0$ and $\lambda=2.5$.

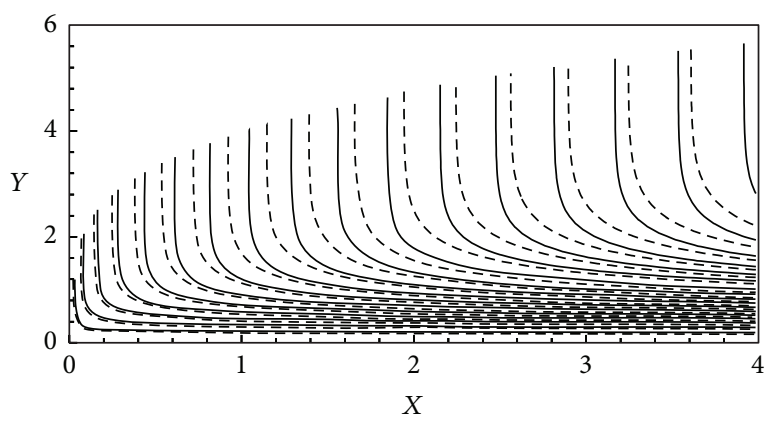

(a)

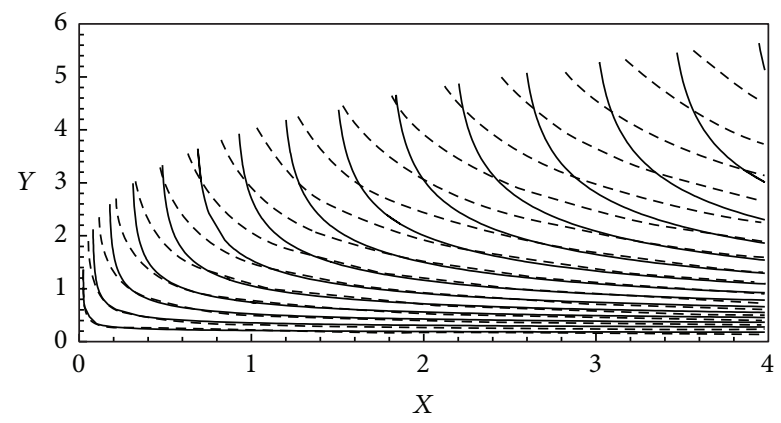

(b)

FIGURE 6: Streamlines (a) solid for $\varepsilon=0.0$ and dashed for $\varepsilon=1.0$ and (b) solid for $\varepsilon=3.0$ and dashed for $\varepsilon=5.0$ while $\mathrm{Ra}=100.0$ and $\lambda=2.5$.

\section{Results and Discussion}

Equations (10)-(12) subject to the boundary conditions (13a) and (13b) are solved numerically using marching order implicit finite-difference method. The numerical solutions are started at $X=0.0$ and proceeded to the downstream region. Here it should be mentioned that the solutions of the parabolic equations (10)-(12) can be terminated anywhere at $X>0$. In this investigation we have taken solutions up to $X=$ 4. Solutions are obtained for the Rayleigh number Ra (50.0, 100.0, and 200.0), heat generation parameter $\lambda(=0.0,2.5$, 5.0 ), and for a wide range of values of the variable viscosityvariation parameter $\varepsilon(=-2.0,-1.0,0.0,1.0,2.0,3.0$, and 5.0 ). Numerical values of the shearing stress in terms of the 


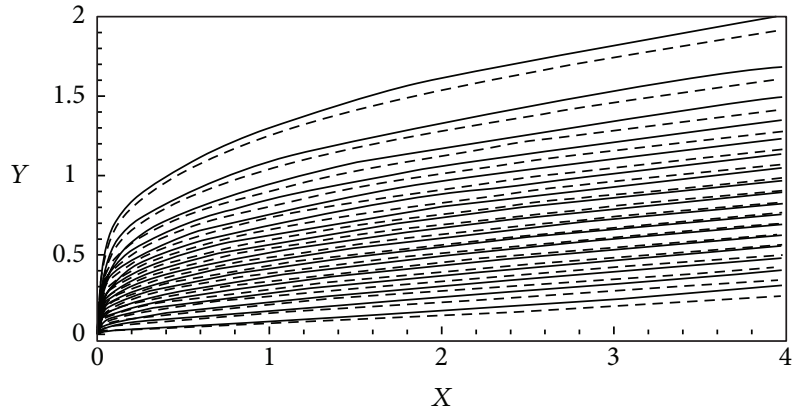

(a)

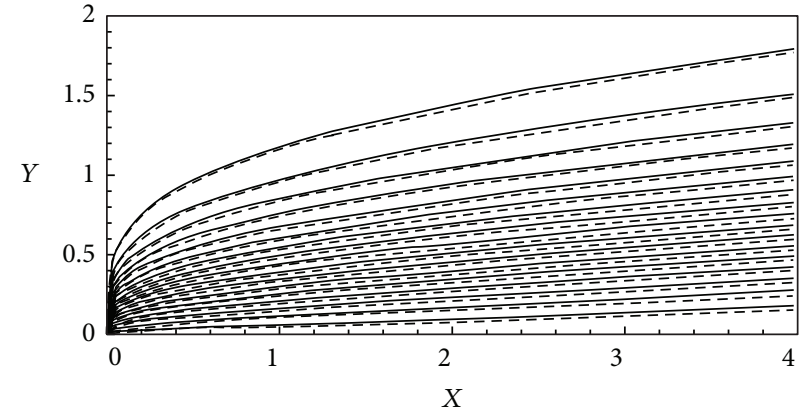

(b)

Figure 7: Isotherms (a) solid for $\varepsilon=0.0$ and dashed for $\varepsilon=1.0$ and (b) solid for $\varepsilon=3.0$ and dashed for $\varepsilon=5.0$ while $\mathrm{Ra}=100.0$ and $\lambda=2.5$.

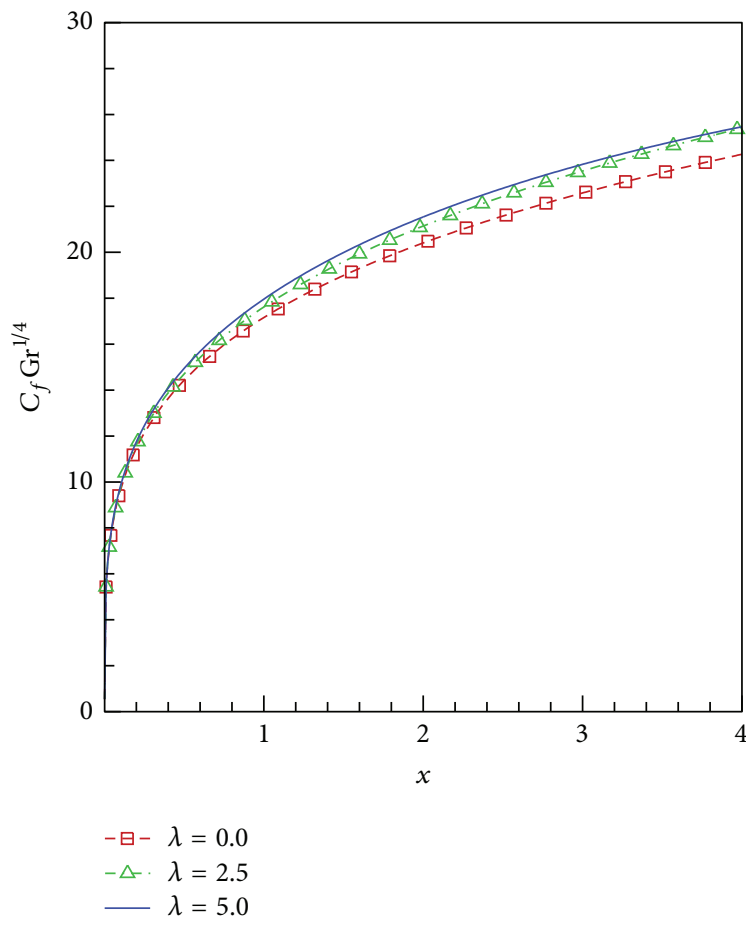

(a)

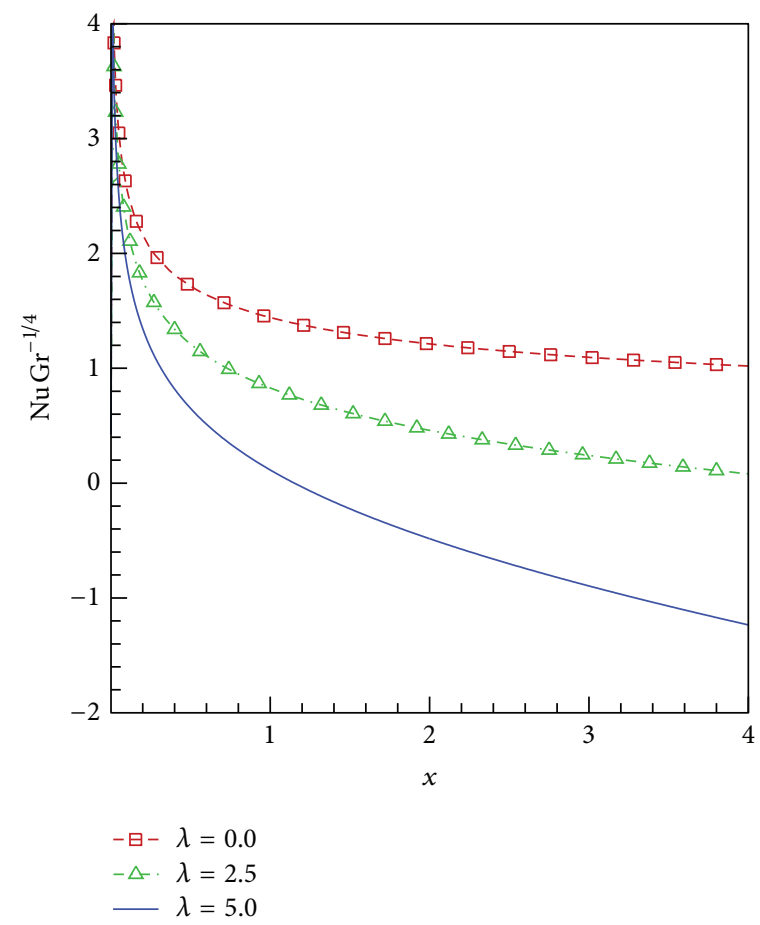

(b)

FiguRE 8: (a) Skin-friction coefficient. (b) Rate of heat transfer for different values of $\lambda$ while $\mathrm{Ra}=100.0$ and $\varepsilon=3.0$.

local skin-friction coefficient $C_{f} \mathrm{Gr}^{1 / 4}$ from (15) and the heat transfer rate in terms of the local Nusselt number $\mathrm{Nu} \mathrm{Gr}^{-1 / 4}$ from (16) are calculated. Numerical values of $C_{f} \mathrm{Gr}^{1 / 4}$ and $\mathrm{Nu} \mathrm{Gr}^{-1 / 4}$ are depicted in Figures 2-4, 8, and 10. For the whole computations $\operatorname{Pr}$ is fixed at 1 .

In order to validate our numerical results the comparison with the published results is not straightforward, because the present boundary layer formulation is a little bit different from the available literature. For comparison, following Ede [29], it has been assumed $\mathrm{Ra}=1$ in (11) and (1/Pr) accompanied with the diffusion term $\left(\partial^{2} \theta / \partial Y^{2}\right)$ of (12). For $\varepsilon=\lambda=0.0$, the numerical results of the modified Nusselt number $\mathrm{Nu}(\mathrm{Gr} / X)^{-1 / 4}$ are presented in Table 1 for the three Prandtl numbers along with the results of Ede [29] and Huang et al. [30]. The comparison shows that the present results obtained using marching order implicit finite difference method are in an excellent agreement with the solutions of Ede [29] and Huang et al. [30]. A grid independence test has been conducted for three different grid arrangements as Case 1: $200 \times 1000(x \times y)$, Case 2: $400 \times 2000$, and Case 3: $800 \times 4000$ which is shown in Figure 2. The results are shown in terms of the local skin-friction coefficient as well as the local Nusselt number and the agreement between the results is quite excellent. The whole simulation has been done using the grid arrangement as in Case 2.

Figure 3 shows the effect of the viscosity-variation parameter $\varepsilon(=-2.0,-1.0,0.0,1.0$, and 2.0$)$ on the local skinfriction coefficient $C_{f} \mathrm{Gr}^{1 / 4}$ and the local rate of heat transfer $\mathrm{Nu} \mathrm{Gr}{ }^{-1 / 4}$ without having any heat generation. For $\varepsilon<0.0$, 

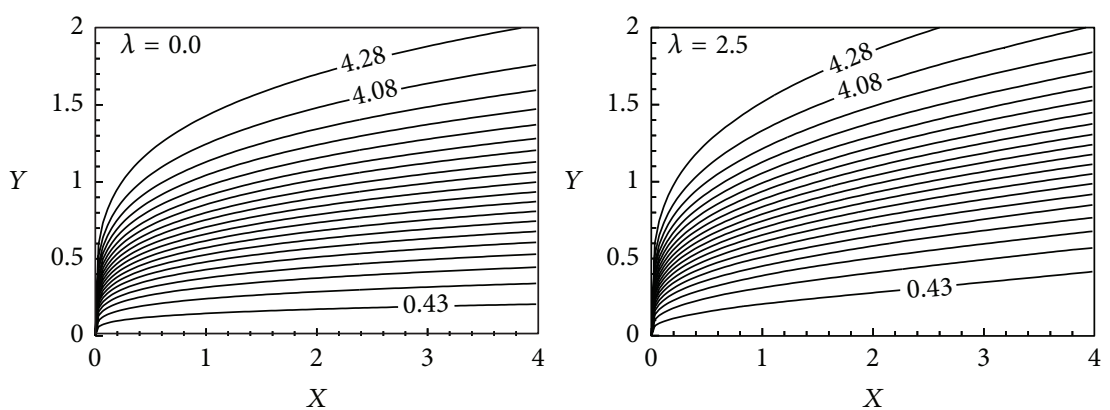

(a)
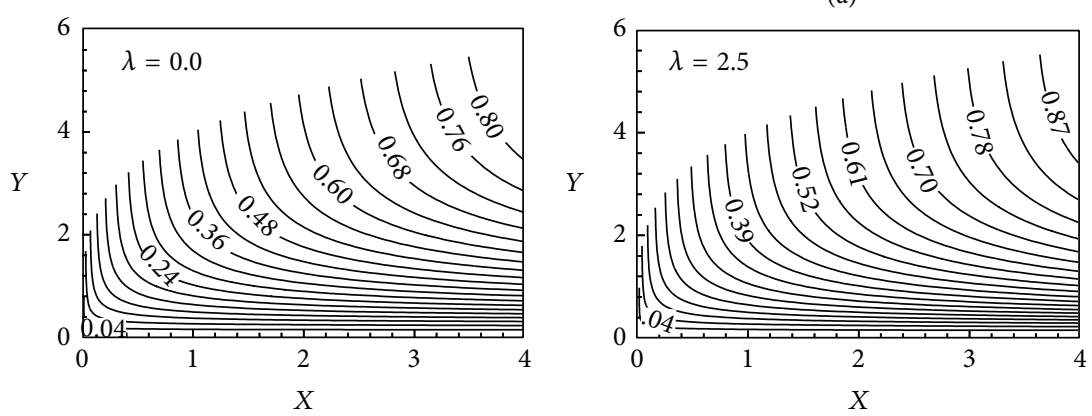

(b)
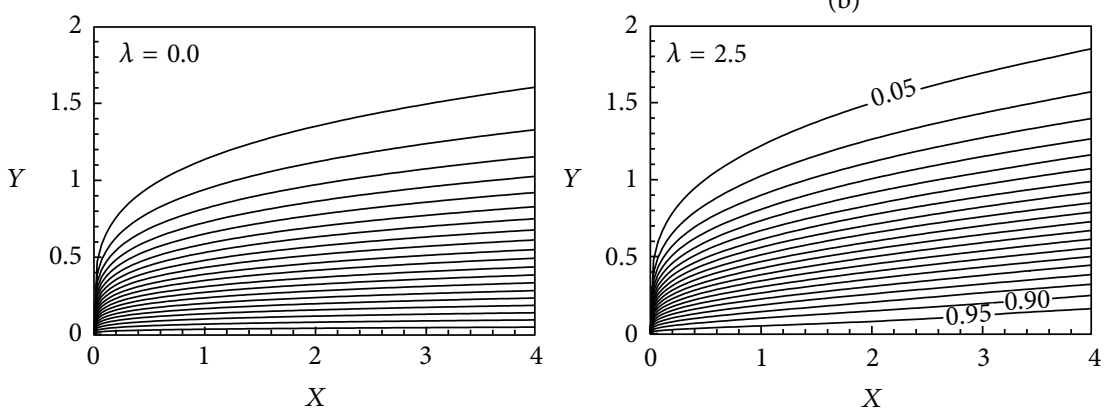
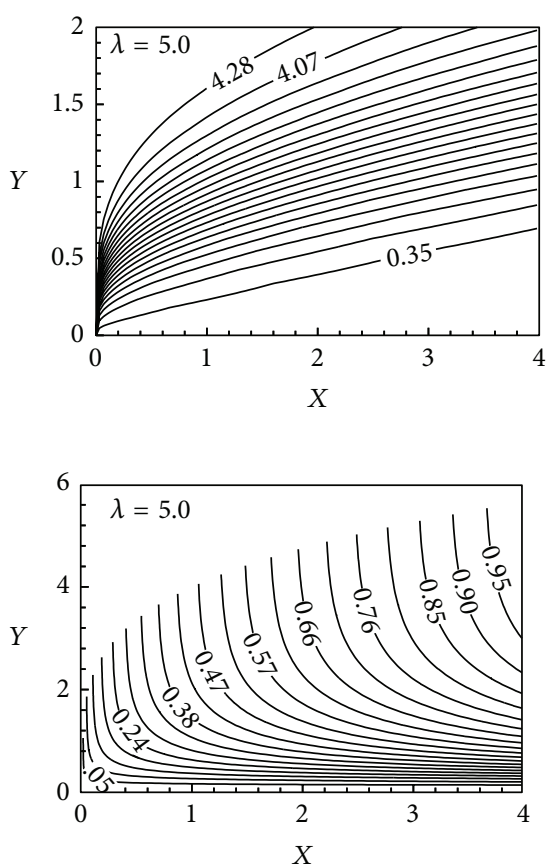

(c)

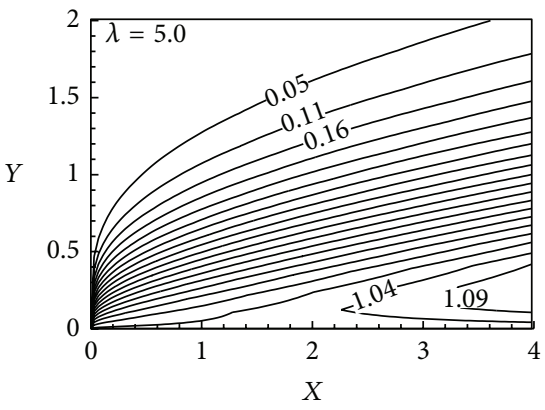

Figure 9: Isolines viscosity (a), streamlines (b), and isotherms (c) for different values of $\lambda$ while $\mathrm{Ra}=100.0$ and $\varepsilon=3.0$.

TABLE 1: Comparison of the Nusselt number $\mathrm{Nu}(\mathrm{Gr} / X)^{-1 / 4}$ with Ede [29] and Huang et al. [30].

\begin{tabular}{lccc}
\hline $\operatorname{Pr}$ & Ede [29] & Huang et al. [30] & Present results \\
\hline 10 & 0.8269 & 0.8268 & 0.82683 \\
100 & 1.5506 & 1.5486 & 1.54946 \\
1000 & 2.8047 & 2.8084 & 2.80356 \\
\hline
\end{tabular}

the local skin-friction coefficient increases and the local Nusselt number decreases. Because, for $\varepsilon<0.0$, the kinematic viscosity of the fluid increases this increases wall shear stress. On the other hand, for $\varepsilon>0.0$, the local skin-friction coefficient decreases and the local Nusselt number increases due to small kinematic viscosities of the fluid. For $\varepsilon \gg 0.0$, the kinematic viscosities are very small which produce zero wall shearing stress. This is an impractical situation to get an inviscid boundary layer. In the present investigation for $\varepsilon>5$, the values of the kinematic viscosity are approximately zero. For $\varepsilon \ll 0.0$, the kinematic viscosities are so high which are appropriate for the highly non-Newtonian shear-thickening fluids.

The effect of the viscosity-variation parameter, $\varepsilon(=0.0$, 1.0, 3.0, and 5.0) having heat generation effects $\lambda(=2.5)$ on the local skin-friction coefficient $C_{f} \mathrm{Gr}^{1 / 4}$ and the local rate of heat transfer $\mathrm{Nu} \mathrm{Gr}^{-1 / 4}$, is illustrated in Figures 4(a)4 (b), respectively, while $\mathrm{Ra}=100.0$. From these figures it is seen that the skin-friction coefficient decreases and the rate of heat transfer increases while the viscosity-variation parameter increases. It is happening due to the fact that neat surface viscosity of the fluid increases and, for high viscous fluid, the velocity gradient decreases and hence the skinfriction coefficient decreases. For high viscosity of the fluid, the temperature distribution decreases within the boundary layer (see Figure 7). Owing to the temperature difference between the plate and fluid, the rate of heat transfer increases significantly. For example at $X=2.0$, the local Nusselt number $\mathrm{Nu} \mathrm{Gr}^{-1 / 4}$ increase $183.12 \%$ as increasing values of $\varepsilon$ from 0 to 5 . 


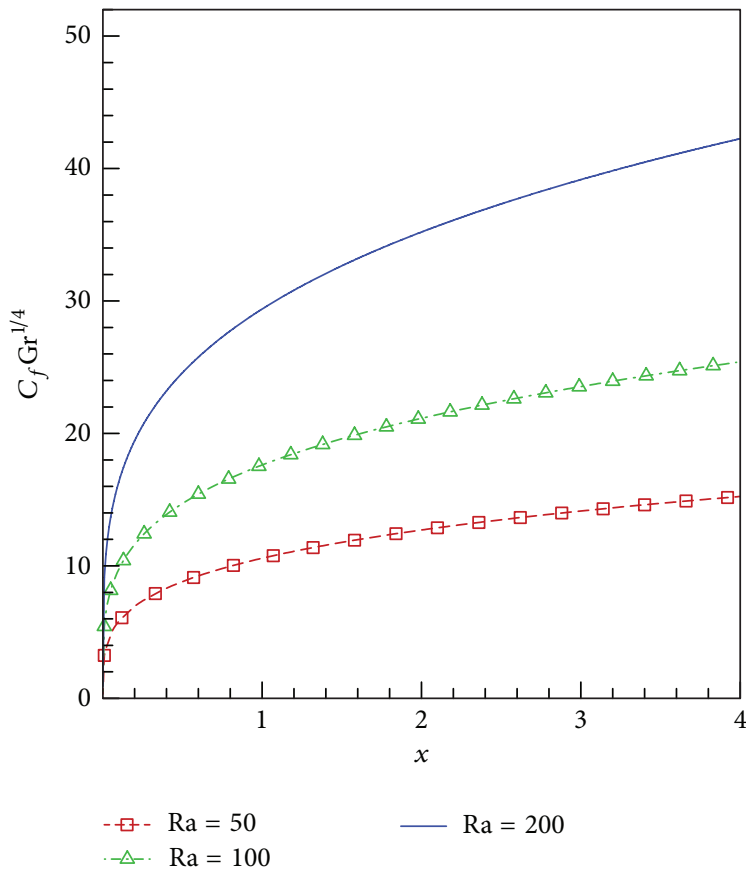

(a)

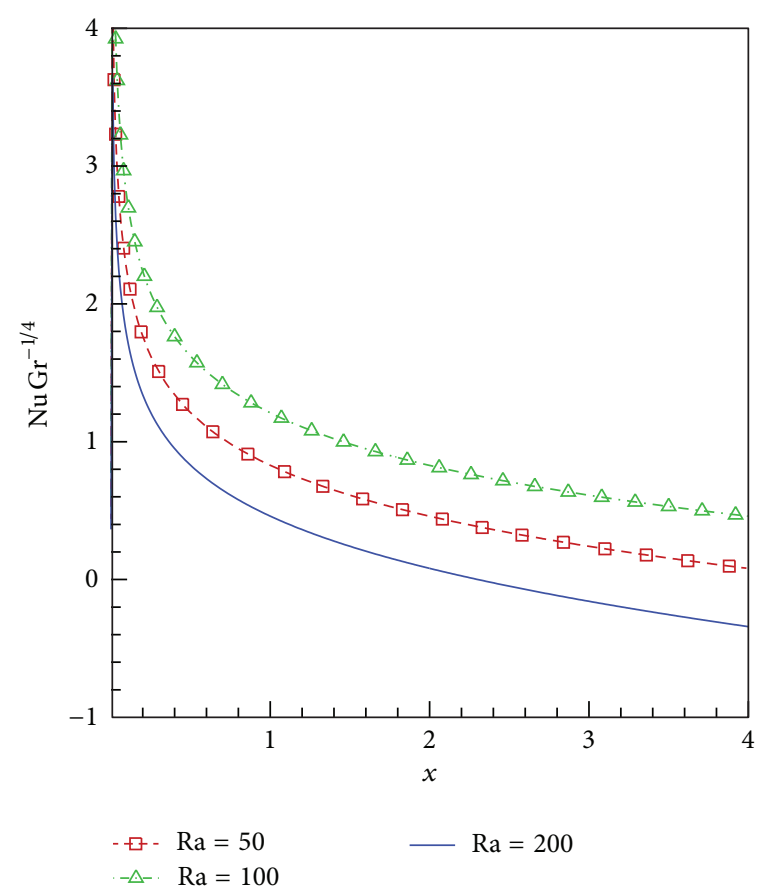

(b)

Figure 10: (a) Skin-friction coefficient. (b) Rate of heat transfer for different values of Ra while $\lambda=2.5$ and $\varepsilon=3.0$.

Figures 5-7 show the isolines viscosity, streamlines, and isotherms, respectively, for different values of the viscosityvariation parameter while $\mathrm{Ra}=100.0$ and $\lambda=2.5$. From Figures 5(a)-5(c), it was clearly seen that the viscosity of the fluid increased at the vicinity of the surface which indicates that the viscosity of the fluid is strongly dependent on temperature. Due to high viscosity of the fluid, the velocity distribution decreases within the boundary layer and hence the values of the stream function $\Psi$ decrease which are shown by the dashed lines in Figure 6. Also the temperature distribution reduces slightly for large values of $\varepsilon$. Finally, it can be concluded that the momentum and thermal boundary layer become thin for high viscous fluid and which is expected.

Figures 8(a)-8(b) show the local skin-friction coefficient $C_{f} \mathrm{Gr}^{1 / 4}$ and the local rate of heat transfer $\mathrm{NuGr}^{-1 / 4}$ for the different values of the heat generation parameter $\lambda(=0.0,2.5$, and 5.0) while $\mathrm{Ra}=100.0$ and $\varepsilon=3.0$. From these figures, it is seen that the increase of heat generation parameter $\lambda$ leads to decreasing the local skin-friction coefficient $C_{f} \mathrm{Gr}^{1 / 4}$ and the local Nusselt number $\mathrm{Nu} \mathrm{Gr}^{-1 / 4}$. These are expected, since the heat generation mechanism creates a layer of hot fluid near the surface and finally the resultant temperature of the fluid exceeds the surface temperature (see isotherms in Figure 9 for $\lambda=5.0$ ). For this reason the rate of heat transfer from the surface decreases. Owing to enhanced temperature, viscosity of the fluid increases and the corresponding local skinfriction coefficient decreases slightly, which is also expected.

Figure 9 illustrates the effect of the heat generation parameter $\lambda$ on the development of isolines viscosity (a), streamlines (b), and isotherms (c), which are plotted for $\mathrm{Ra}=$ 100.0 and $\varepsilon=3.0$. For large heat generation parameter the viscosity of the fluid increases which are shown in the figures of the isolines of viscosity. From Figure 9(b), it is seen that without effect of heat generation (i.e., $\lambda=0.0$ ) the nondimensional value of $\Psi_{\max }$ within the computational domain is about 0.8 when the boundary layer thickness is the highest, but $\Psi_{\max }$ increases with the increment of $\lambda$ and it attains about 0.87 and 0.95 for $\lambda=2.5$ and 5.0, respectively. This phenomenon fully coincides with the early discussion made on Figure 8(a); the fluid speeds up as $\lambda$ increases and the thickness of the velocity boundary layer also grows. The isotherm patterns for corresponding values of $\lambda$ are shown in Figure 9(c). From all these fames, it is seen that the growth of thermal boundary layer over the surface of the flat plate is significant. As $X$ increases from the leading edge $(X \approx 0.0)$, the hot fluid raises and hence the thickness of the thermal boundary layer increases. But this phenomenon is not very straightforward as can be seen in 2nd and 3rd frames of Figure 9(c) for $\lambda=2.5$ and for $\lambda=5.0$ that the levels of isotherm are noticeably higher than the surface level, and in 3 rd frame the fluid temperature exceeds the surface level and the surface heat transfer rate became negative which was also noticed in Figure 8(b).

The effect of different values of the Rayleigh Ra on skin friction coefficient $C_{f} \mathrm{Gr}^{1 / 4}$ and the rate of heat transfer $\mathrm{Nu} \mathrm{Gr}^{-1 / 4}$ with the heat generation parameter $\lambda=2.5$ and for $\varepsilon=3.0$ are illustrated in Figures 10(a)-10(b), respectively. It can easily be seen that the effect of the Rayleigh number $\mathrm{Ra}$ leads to increasing the skin friction coefficient $C_{f} \mathrm{Gr}^{1 / 4}$ 


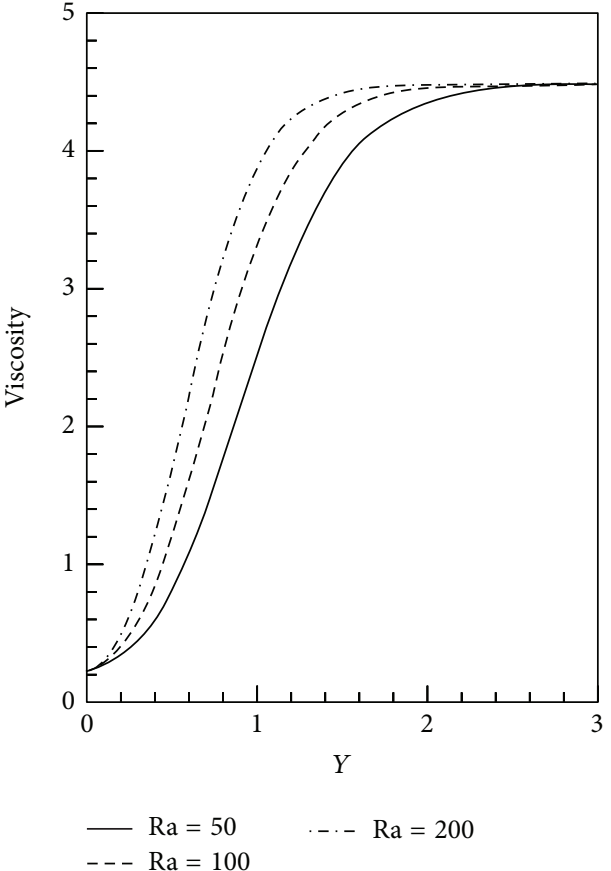

(a)

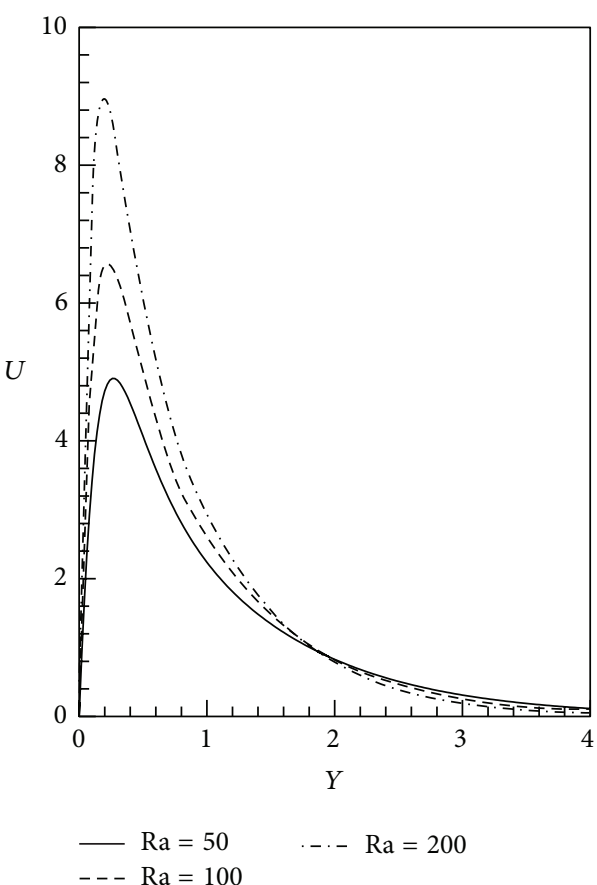

(b)

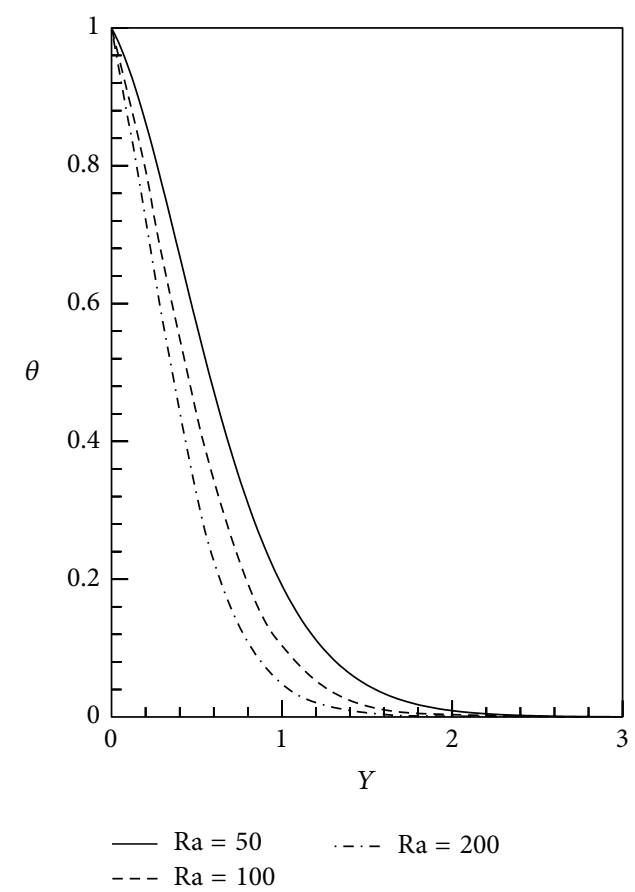

(c)

Figure 11: (a) Viscosity, (b) velocity, and (c) temperature profiles for different values of Ra while $\lambda=2.5$ and $\varepsilon=3.0$ at $X=1.0$.

and the rate of heat transfer $\mathrm{Nu} \mathrm{Gr}^{-1 / 4}$. This phenomenon can easily be understood from the fact that with the increasing values of the Rayleigh number Ra, the buoyancy force increase, which speeds up the fluid velocity (see Figure 11(b)), that means, increases the velocity gradient and hence the local skin-friction coefficient $C_{f} \mathrm{Gr}^{1 / 4}$ enhances. Owing to the increase in the values of Ra leads to decrease the fluid temperature (see in Figure 11(c)) within the boundary layer and the associated thermal boundary layer becomes thinner. For decreasing fluid temperature, the temperature difference between fluid and surface increases and the corresponding rate of heat transfer $\mathrm{Nu} \mathrm{Gr}^{-1 / 4}$ increases. 


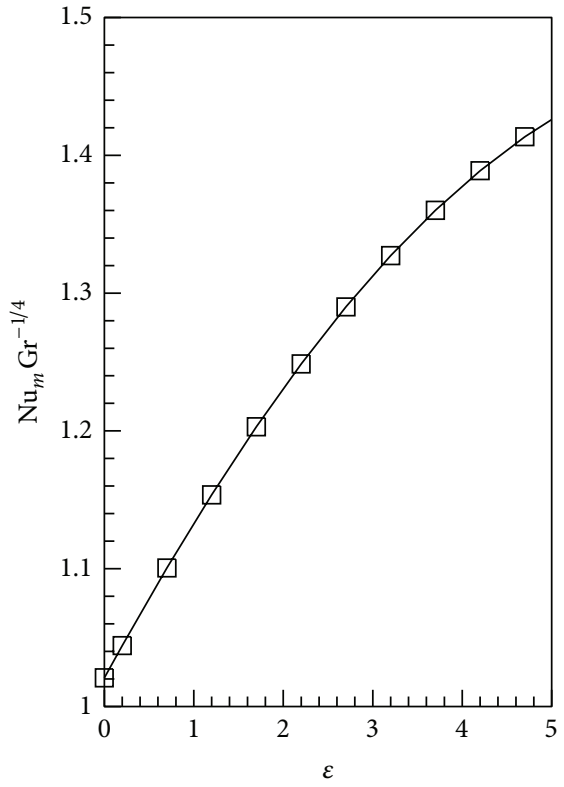

(a)

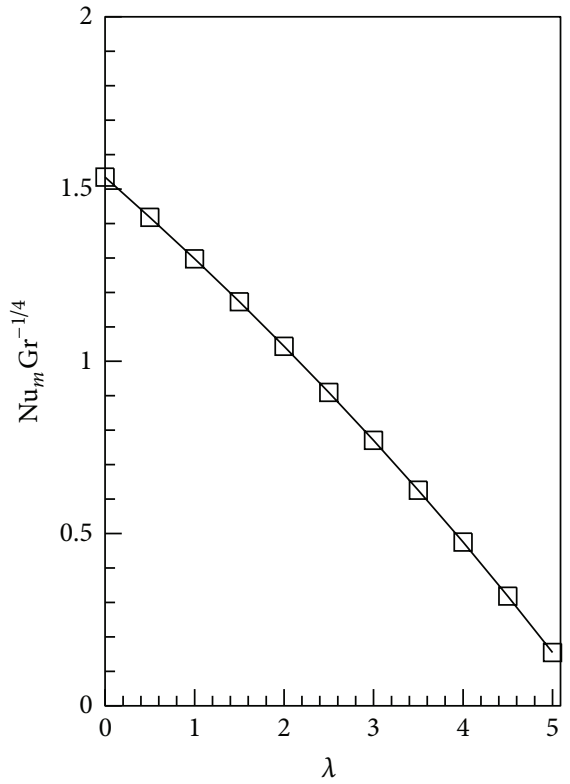

(b)

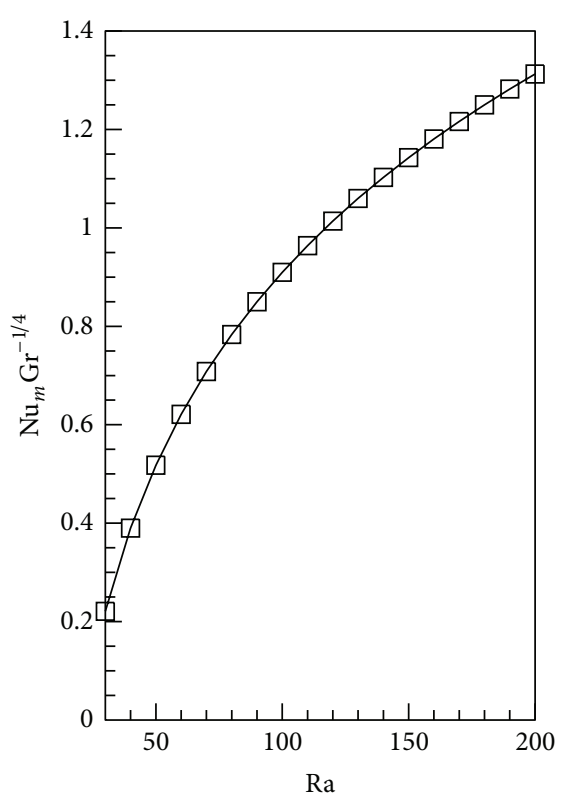

(c)

FIGURE 12: Average Nusselt number for (a) viscosity-variation parameter while $\mathrm{Ra}=200$ and $\lambda=2.5$ (b) heat generation parameter while Ra $=100$ and $\varepsilon=3.0$ (c) Rayleigh number while $\lambda=2.5$ and $\varepsilon=3.0$.

Figures 12(a)-12(c) depict the average rate of heat transfer in terms of the average Nusselt number with the effect of the viscosity variation, Rayleigh number, and the heat generation, respectively. Average Nusselt number increases with the increase of viscosity-variation parameter and the Rayleigh number. On the other hand, with increase of heat generation the average Nusselt number decrease like local Nusselt number.

\section{Conclusion}

Numerical solutions for the steady laminar free convection boundary layer flow along a vertical flat plate subjected to a uniform surface temperature in presence of heat generation effect with fluid having viscosity which is the exponential function of temperature have been investigated theoretically. The governing boundary layer equations of motion are transformed into a nondimensional form and the resulting nonlinear systems of partial differential equations are reduced to convenient boundary layer equations, which are solved numerically by using marching order implicit finite difference method. From the present investigation the following conclusions may be drawn.

(i) With effect of viscosity-variation parameter $\varepsilon$ and the Rayleigh number Ra, the skin-friction coefficient decreases and the local and average rate of heat transfer increase.

(ii) An increase in values of $\lambda$ leads to slightly decreasing the skin-friction coefficient $C_{f} \mathrm{Gr}^{1 / 4}$ but decrease of the local and average rate of heat transfer is more significant. (iii) For increase values of viscosity-variation parameter $\varepsilon$, the momentum and thermal boundary layer become thinner.

(iv) With the effect of the Rayleigh number Ra both the viscosity and velocity distribution increase and the temperature distributions decrease significantly and the thickness of the momentum boundary layer is enhanced.

\section{Nomenclature}

$C_{f}: \quad$ Local skin-friction coefficient

$C_{p}$ : Specific heat at constant pressure

Gr: Grashof number

$L: \quad$ Reference length of the plete

Nu: Local Nusselt number

Pr: Prandtl number

$q^{\prime \prime}$ : Volumetric rate of heat generation

$Q_{0}$ : Heat generation constant

Ra: Rayleigh number

$T: \quad$ Dimensional temperature of the fluid

$T_{\infty}:$ Ambient temperature

$T_{w}$ : Wall temperature

$\bar{u}, \bar{v}$ : Velocity components along the $\bar{x}, \bar{y}$ axes, respectively

$U, V$ : Dimensionless fluid velocities in the $X, Y$ directions, respectively

$\bar{x}, \bar{y}$ : Cartesian coordinate measured along the plate and normal to it, respectively

$X: \quad$ Axial direction along the plate

$Y$ : Pseudosimilarity variable. 


\section{Greek Symbols}

$\alpha$ : Thermal diffusivity

$\beta$ : Thermal expansion coefficient

$\varepsilon$ : Viscosity-variation parameter

$\lambda$ : Heat generation parameter

$\nu$ : Kinematic viscosity

$v_{0}$ : Reference kinematic viscosity

$\theta:$ Dimensionless temperature of the fluid

$\rho: \quad$ Fluid density

$\tau_{w}:$ Shear stress

$\Pi$ : Nondimensional viscosity

$\delta_{M}$ : Momentum boundary layer thickness

$\delta_{T}$ : Thermal boundary layer thickness.

\section{Conflict of Interests}

The authors declare that there is no conflict of interests regarding the publication of this paper.

\section{References}

[1] K. Vajravelu and A. Hadjinicolaou, "Heat transfer in a viscous fluid over a stretching sheet with viscous dissipation and internal heat generation," International Communications in Heat and Mass Transfer, vol. 20, no. 3, pp. 417-430, 1993.

[2] M. M. Molla, M. A. Hossain, and L. S. Yao, "Natural convection flow along a vertical wavy surface with uniform surface temperature in presence of heat generation/absorption," International Journal of Thermal Sciences, vol. 43, no. 2, pp. 157-163, 2004.

[3] M. M. Molla, M. A. Taher, M. K. Chowdhury, and M. A. Hossain, "Magnetohydrody-namic natural convection flow on a sphere in presence of heat generation," Nonlinear Analysis: Modelling and Control, vol. 10, no. 4, pp. 349-363, 2005.

[4] M. M. Molla, M. A. Hossain, and M. A. Taher, "Magnetohydrodynamic natural convection flow on a sphere with uniform heat flux in presence of heat generation," Acta Mechanica, vol. 186, no. 1-4, pp. 73-86, 2006.

[5] M. M. Molla, M. A. Hossain, and M. C. Paul, "Natural convection flow from an isothermal horizontal circular cylinder in presence of heat generation," International Journal of Engineering Science, vol. 44, no. 13-14, pp. 949-958, 2006.

[6] T. Cebeci and P. Bradshaw, Physical and Computational Aspects of Convective Heat Transfer, Springer, New York, NY, USA, 1984.

[7] J. Gary, D. R. Kassoy, H. Tadjeran, and A. Zebib, “The effect of significant viscosity variation on convective heat transport in water-saturated porous media," Journal of Fluid Mechanics, vol. 117, pp. 233-249, 1982.

[8] K. N. Mehta and S. Sood, "Transient free convection flow with temperature dependent viscosity in a fluid saturated porous medium," International Journal of Engineering Science, vol. 30, no. 8, pp. 1083-1087, 1992.

[9] F. M. Hady, A. Y. Bakier, and R. S. R. Gorla, "Mixed convection boundary layer flow on a continuous flat plate with variable viscosity," Heat and Mass Transfer, vol. 31, pp. 169-172, 1996.

[10] N. G. Kafoussias and E. W. Williams, "The effect of temperaturedependent viscosity on free-forced convective laminar boundary layer flow past a vertical isothermal flat plate," Acta Mechanica, vol. 110, no. 1-4, pp. 123-137, 1995.

[11] N. G. Kafoussias, D. A. S. Rees, and J. E. Daskalakis, "Numerical study of the combined free-forced convective laminar boundary layer flow past a vertical isothermal flat plate with temperaturedependent viscosity," Acta Mechanica, vol. 127, no. 1-4, pp. 3950, 1998.

[12] M. A. Hossain, M. S. Munir, and I. Pop, "Natural convection flow of a viscous fluid with viscosity inversely proportional to linear function of temperature from a vertical wavy cone," International Journal of Thermal Sciences, vol. 40, no. 4, pp. 366371,2001

[13] A. Hossain, S. Munir, and D. A. S. Rees, "Flow of viscous incompressible fluid with temperature dependent viscosity and thermal conductivity past a permeable wedge with uniform surface heat flux," International Journal of Thermal Sciences, vol. 39, no. 6, pp. 635-644, 2000.

[14] M. M. Molla, M. A. Hossain, and R. S. R. Gorla, "Natural convection flow from an isothermal horizontal circular cylinder with temperature dependent viscosity," Heat and Mass Transfer, vol. 41, no. 7, pp. 594-598, 2005.

[15] J. X. Ling and A. Dybbs, "Forced convection over a flat plate submersed in a porous medium: variable viscosity case," Paper 87-WA/HT-23, ASME, New York, NY, USA, 1987.

[16] J. Charraudeau, "Influence of physical properties of gradients in forced convection-application in the case of the tube," International Journal of Heat and Mass Transfer, vol. 18, no. 1, pp. 87-95, 1975.

[17] M. M. Molla and M. A. Hossain, "Effects of chemical reaction, heat and mass diffusion in natural convection flow from an isothermal sphere with temperature dependent viscosity," Engineering Computations, vol. 23, no. 7, pp. 840-857, 2006.

[18] M. M. Molla, S. C. Saha, and M. A. I. Khan, "MHD natural convection flow from an isothermal horizontal circular cylinder under consideration of temperature dependent vis-cosity," Engineering Computations, vol. 29, 8, pp. 875-887, 2012.

[19] D. D. Gray and A. Giorgini, "The validity of the boussinesq approximation for liquids and gases," International Journal of Heat and Mass Transfer, vol. 19, no. 5, pp. 545-551, 1976.

[20] Q. Wang, H. Yoo, and Y. Jaluria, "Convection in a horizontal rectangular duct under constant and variable property formulations," International Journal of Heat and Mass Transfer, vol. 46, no. 2, pp. 297-310, 2003.

[21] S. C. Saha, Y. T. Gu, M. M. Molla, S. Siddiqa, and M. A. Hossain, "Natural convection from a vertical plate embedded in a stratified medium with uniform heat source," De-Salination and Water Treatment, vol. 44, no. 1-3, pp. 7-14, 2012.

[22] T. Kao, "Locally non-similar solution for laminar free convection adjacent to a ver-tical wall," ASME, Journal of Heat Transfer, vol. 98, pp. 321-322, 1976.

[23] N. T. Wright and B. Gebhart, "The entrainment flow adjacent to an isothermal vertical surface," International Journal of Heat and Mass Transfer, vol. 37, no. 1, pp. 213-231, 1994.

[24] K. E. Torrance and D. L. Turcotte, "Thermal convection with large viscosity variations," Journal of Fluid Mechanics, vol. 47, pp. 113-125, 1971.

[25] J. R. Booker, "Thermal convection with strongly temperature dependent viscosity," Journal of Fluid Mechanics, vol. 76, pp. 741-754, 1976.

[26] K. C. Stengel, D. S. Oliver, and J. R. Booker, "Onset of convection in a variable viscos-ity fluid," Journal of Fluid Mechanics, vol. 120, pp. 411-431, 1982.

[27] F. M. Richter, H. C. Nataf, and S. F. Daly, "Heat transfer and horizontally averaged temperature of convection with large viscosity variations," Journal of Fluid Mechanics, vol. 129, pp. 173-192, 1983. 
[28] A. Bottaro, P. Metzener, and M. Matalon, "Onset and twodimensional patterns of convection with strongly temperaturedependent viscosity," Physics of Fluids A, vol. 4, no. 4, pp. 655$663,1992$.

[29] A. J. Ede, "Advances in free convection," Advances in Heat Transfer, vol. 4, pp. 1-64, 1967.

[30] M. J. Huang, J. S. Huang, Y. L. Chou, and C. K. Chen, "Effects of Prandtl number on free convection heat transfer from a vertical plate to a non-Newtonian fluid," Journal of Heat Transfer, vol. 111, no. 1, pp. 189-191, 1989. 

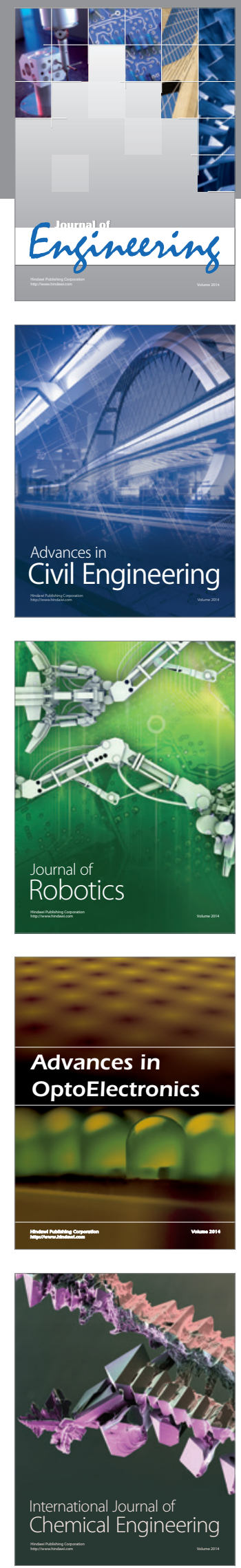

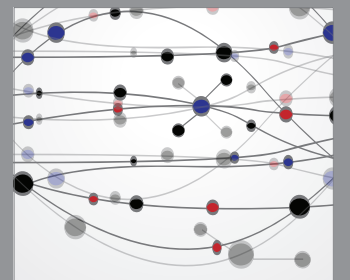

The Scientific World Journal
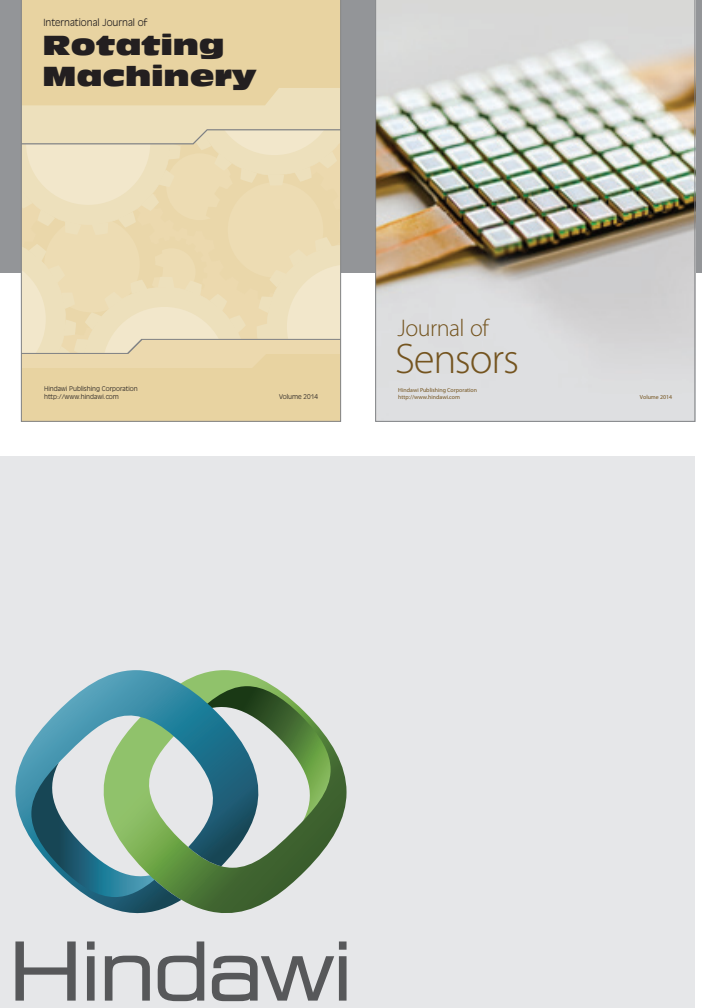

Submit your manuscripts at http://www.hindawi.com
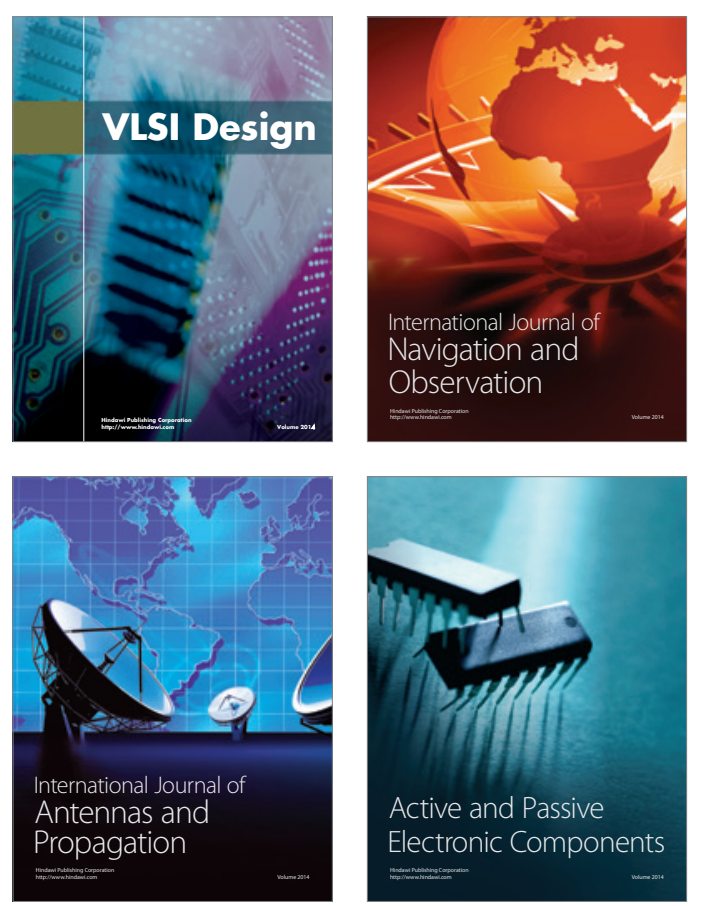
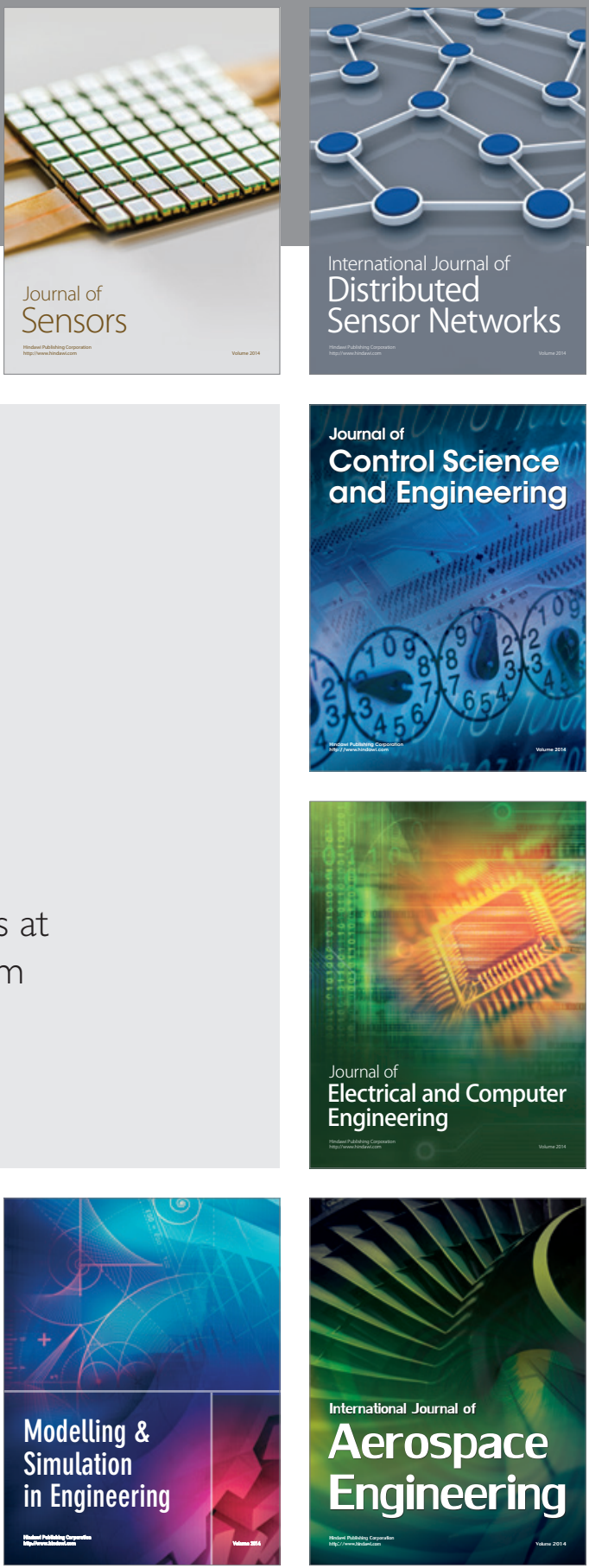

Journal of

Control Science

and Engineering
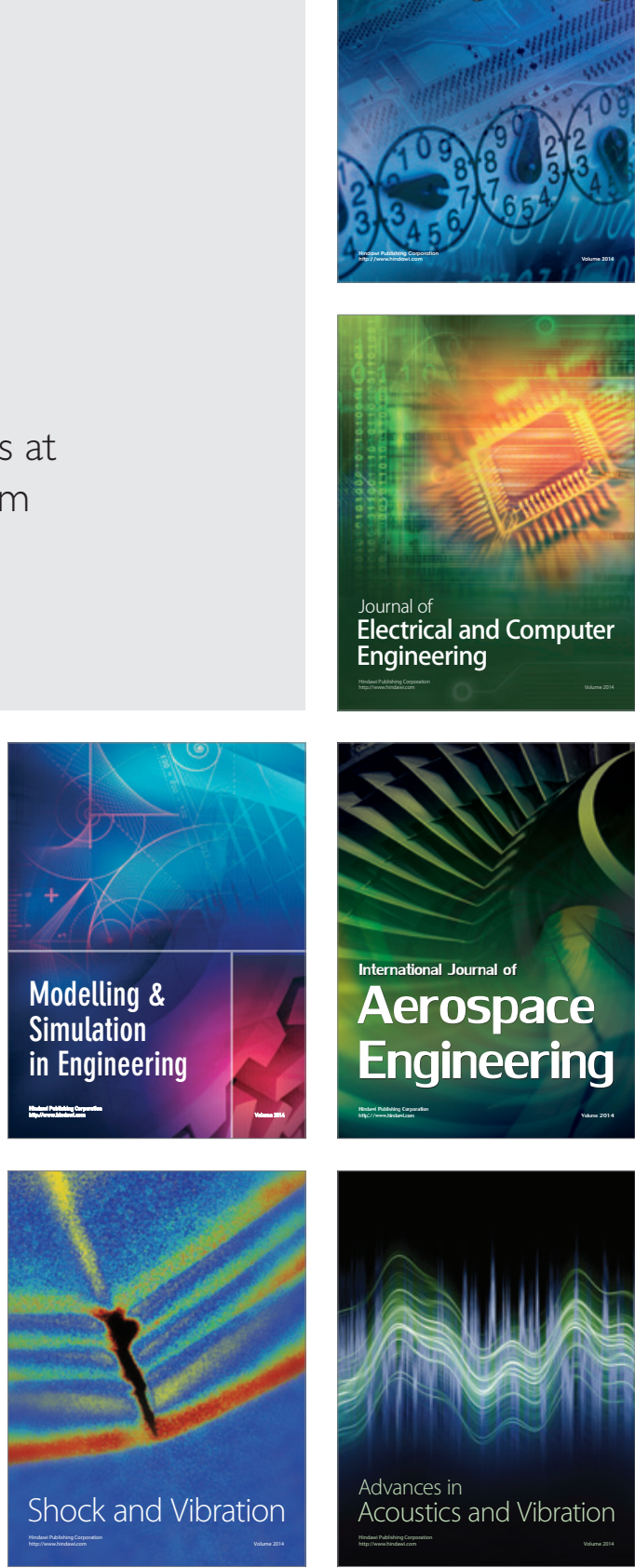